\title{
An Automatic Brain Tumor Detection and Segmentation using Hybrid Method
}

\author{
Sreedhanya S. \\ Computer Engineering, \\ Datta Meghe College of Engineering, \\ Mumbai University, Airoli
}

\author{
Chhaya S. Pawar \\ Asst. prof. Dept. of Computer Engineering, \\ Datta Meghe College of Engineering, \\ Mumbai University, Airoli
}

\begin{abstract}
In the field of medical image processing, brain tumor detection and segmentation using MRI scan has become one of the most important and challenging research areas. In which manual detection and segmentation of brain tumors using brain MRI scan forms a large part of human intervention for detection and segmentation taken per patient, is both tedious and has huge internal and external observer detection and segmentation variability. Hence, there is high demand for an automatic brain tumor detection and segmentation using brain MR images to overcome manual segmentation. So in current days a number of methods have proposed by researchers. But still there is no complete automated system developed yet, is due to accuracy and robustness issues. So, this paper provides a review of the methods and techniques that used to detect and segment brain tumor through MRI segmentation. Finally, the paper concludes with one of the efficient hybrid method which shows high accuracy on detection of brain tumor with proposed Gaussian Mixture Model (GMM).
\end{abstract}

\section{Keywords}

Brain Tumor, MRI, Tumor Segmentation and Detection, FHNN, GMM

\section{INTRODUCTION}

Brain tumor, which is one of the leading brain diseases, has affected and destroyed many lives. According to International Agency for Research on Cancer (IARC) it is estimated more than people diagnosed for brain tumor per year around the world with more than mortality [11]. The statistics show the low survival rate of brain tumor patients even though the brain tumor disease has been the center of attention of thousands of researchers for many decades, around the world. In recent years, researchers from different disciplines such as medical and computer field have integrated their knowledge and efforts to better understand the brain disease and further treatment.

Magnetic resonance (MR) imaging and computed tomography (CT) scan of the head are the most common tests taken to confirm presence of a brain tumor and identifying its location for selecting treatment options. Currently there is a different treatment option for a brain tumor, which includes surgery, radiation therapy, and chemotherapy. The treatment options depend on several factors, including the size, type and intensity value of the brain. The accurate detection of the type of brain abnormality is highly essential for treatment planning in order to minimize the fatal results.

But segmenting brain tumors is a very difficult task. In the first consideration, there is a large class of tumor types that have a variety of shapes and sizes. The appearance of a brain tumor at different locations in the brain with different image intensities is another factor that makes difficulties in automated brain tumor detection and segmentation. Many techniques have been proposed for the automatic brain tumor detection and segmentation in recent years such as thresholding based, region growing based, clustering based, neural network based, K-means clustering, fuzzy c-means (FCM) and fuzzy c-means strategy is integrated with HNN.

In this paper, suggested a fast c-means based fuzzy Hopfield neural network based method to detect and segment brain tumor boundaries from T1-w and T2-w MR images combined with other image processing techniques to enhancing the brain tumor region extraction and visualization process [5]. For image modeling, Gaussian Mixture Model (GMM) is used [6], to improve the visual quality of different types of input images.

\section{LITERATURE SURVEY}

Many techniques have been proposed to detect the brain tumor in recent years. The proposed methods can be broadly classified into two, intelligent based and non-intelligent based. Most notable intelligent based systems are artificial neural network, fuzzy cmeans, k-means clustering and hybrid methods. The most notable non-intelligent methods include thresholding and region growing. But there is no clear separation between the two, both systems depends one another for getting the best result. Some important methods are discussed in this section.

\subsection{Thresholding Based Methods}

Thresholding based method image segmentation based on gray level intensity value of pixels in the images. A thresholding method has to find an intensity value called the threshold value, which separates the separate classes. The segmentation and classification of classes is based on the grouping of pixels with respect to the intensity values. Some studies are done based on this method, but these papers did not specify the data used for testing and validating their methods.

Shraddha.P.Dhuma and Ashwini.S.Gaikwad [1], they proposed a methodology for fully automated Brain Tumor segmentation from Magnetic Resonance Images using thresholding method. In which an axial slice of T1 weighted MR images have taken with contrast enhancement. This method combines region and edge information to segment brain tumors in MRI. The method is fully automatic and the quality of segmentation in the borders of tumor is relatively good because of using edge information. Image processing system consists of steps like Image preprocessing, Image enhancement, Feature Extraction, Recognition and Identification to get the desired output. 


\subsubsection{Pros and Cons}

The results show good segmentation results and it requires less time because there is no mathematical formula used so this approach reduces a large amount of computation time and shows better accuracy in performance. But expense in terms of computation and large number of MRI scan utilization are the disadvantages.

\subsection{Clustering Techniques}

Clustering is an important method for the segmentation of images to categorize the pixels values with respect to its similarities. Pixels with similar characteristics are grouped or classification of objects into groups according to criteria of the objects referred clusters. In this method, each pixel in the input image assigning to the nearest cluster center. Clustering algorithms are classified as hard clustering (k-means clustering) and soft clustering (fuzzy clustering) etc.

\subsubsection{The K-Means Algorithm}

$\mathrm{K}$-means algorithm is the most well-known clustering technique. The purpose of this algorithm is to minimize the distances of all the elements to their cluster centers. Most of the algorithms in this field are developed by improving kmeans. The algorithm modifies the clusters periodically and runs in a loop until it reaches to optimal solution. The performance of $\mathrm{k}$-means algorithm depending on initial values of cluster centers in clusters. Therefore, the algorithm should be tested for different outcomes with different initial cluster centers by multi-running.

Malathi [2], proposed a tumor detection and segmentation of brain MR images using K-Means clustering. In preprocessing stage, the RGB input image is converted to grayscale image. The preprocessed image is given for image segmentation using K-Means clustering algorithm. The result is shown in Figure 1.
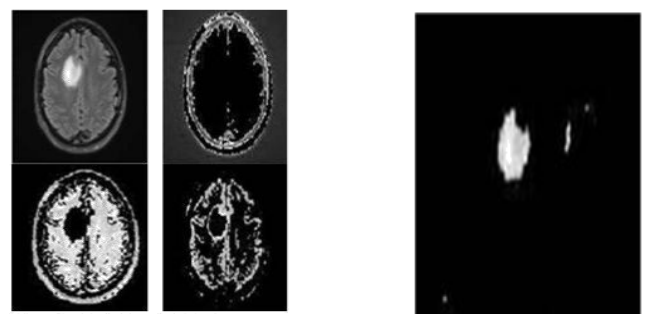

Fig 1: Clustering of MR images and Tumor detected [2]

\subsubsection{Fuzzy Clustering}

Fuzzy clustering is also called as soft clustering. It is one of the forms of clustering technique, in which each data point in pixel can belong to more than one cluster center. It is mainly used in object detection, segmentation and fuzzy modeling. Fuzzy c-means is the one of the widely used algorithm. Clusters can identify through similarity measures. These similarity measures can be chosen based on the data or its application.

Preetha. R and G. R. Suresh [3], they proposed Fuzzy Cmeans algorithm for clustering process. FCM is robust and effective for tumor localization. The Fuzzy C-means clustering with feature extraction and its classification are very hopeful in the field of brain tumor detection and segmentation. The performance of Fuzzy C-means algorithm is calculated and examined based on its segmentation efficiency and convergence rate.

\subsubsection{Pros and Cons}

Shows good accuracy in performance with FCM, segmentation efficiency and convergence rate shows better result. But the number of computation and the complexity is higher because of the data point is belongs to more than one clusters.

\subsection{Hybrid Based Techniques}

Hybrid method combines different methods of machine learning algorithms and make them work together to achieve a better solution to a problem, compared to a single method applying for a same problem. For example Fuzzy c-means method is combined with HNN (Hopfield neural Network) and produces fuzzy Hopfield neural network, this is more effective than other image segmentation. The method used the global histogram of images, in which frequency occurrence of each gray level used to construct the neurons. Consequently, the number of neurons is independent of image size. The experiment result is successful on normal axial brain MR images. But, for the objective functions to converge to a stable state it needs several synchronous iterations.

Abdel-Maksoud, Eman and Mohammed Elmogy[4], they proposed a hybrid method for an efficient image segmentation approach using K-means clustering technique combined with FCM. For getting accurate brain tumor detection, thresholding method and level set segmentation stages are added to it. The performance of image segmentation and detection was evaluated by comparing it with some other segmentation algorithms with respect to accuracy, processing time, and performance as shown in Figure 2. The accuracy was evaluated by comparing the results with the ground truth of each processed image.

\subsubsection{Pros and Cons}

K-means clustering shows minimal computation time and FCM shows good accuracy. But to increase the efficiency of the segmentation process, intensity adjustment process provide more challenging.

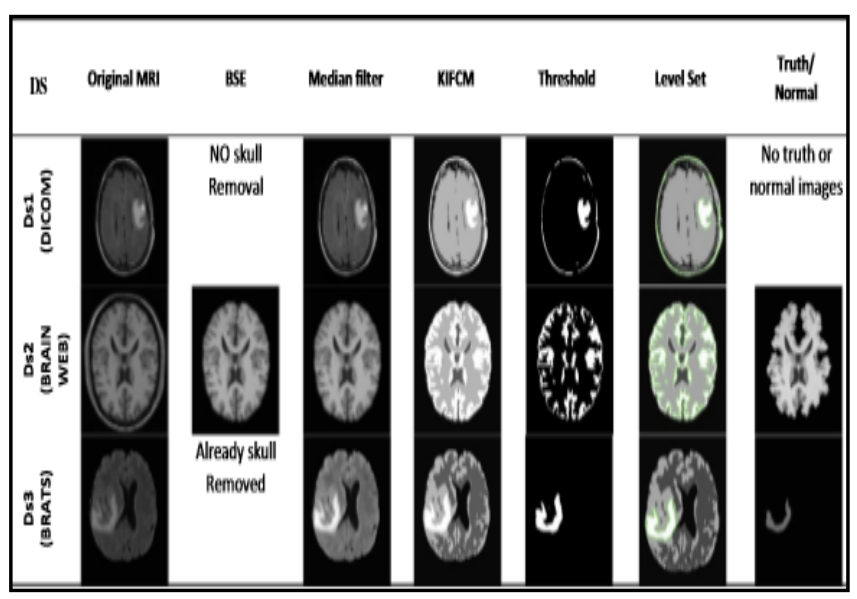

Fig 2: Brain tumor segmentation based on a hybrid clustering technique [4]

\subsection{Guassian mixture model}

The standard methodology in image enhancement for image modelling is Gaussian mixture model. Various fields such as mining of data, processing of information and recognition of 
pattern use GMM. The expectation-maximization (EM) algorithm can use when the number of Gaussians already known. The visual qualities of the input images are very effective and also low contrast images are automatically improved when using the GMM.

Sabahat Fatima and Dr. Shubhangi [6], proposed GMM, In this paper the implementation and analysis of image contrast enhancement using Gaussian mixture model is done by mathematically while comparing with Global Histogram Equalization (GHE). GMM is used for the modification of input images. The equivalent contrast image is developed by changing the pixels grey-levels in the interval of each input image, shown in Figure 3.
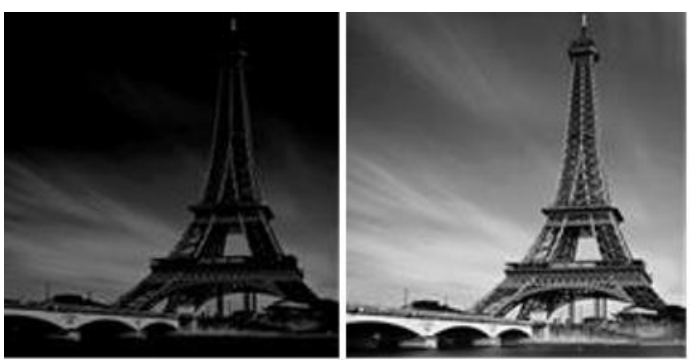

Fig 3: Gray Level Input Image and Equalized Output Image [6]

The main advantages of using GMM algorithm is, it can be applied to both grey-level and color images. The overall content of an input image with high contrast will not be distracted by it. It helps to automatically improves brightness, content and contrast of an image.

\subsection{Hybrid method using FHNN}

Here, discussing an automatic brain tumor detection and segmentation based on fuzzy Hopfield neural network [5] is used. The flow of system is shown in Figure 4. The method introducing three main steps: image preprocessing, tumorous slice detection, and tumor segmentation and visualization.

\subsubsection{Image Preprocessing}

The image preprocessing section performs intensity inhomogeneity correction, background noise removal and skull stripping. The skull stripping method performs the removal of non brain tissues such as: skull and fat from head MRI scan images. In the proposed model, a local entropy minimization with a bicubic spline model introduced, to correct severe intensity in-homogeneity in the MR images. Also, introducing the diffusion filters, used to reduce other noise in the brain MR images.

Otsu's algorithm is used for automatic threshold value selection using, to automatically choose threshold value. Then, binarised image are applied stage by stage to achieve acceptable skull stripped brain images. The skull stripping method has four steps. Initially image binarisation, is performed using threshold value obtained from Otsu's threshold selection algorithm. Morphological operations are the next step to removing the narrow connections from binarised image. Then, the largest connected component from binarised image is selected and then mathematical morphology operations are done. Finally, obtaining the skull stripped brain image using mathematical logical operations on binarised images.

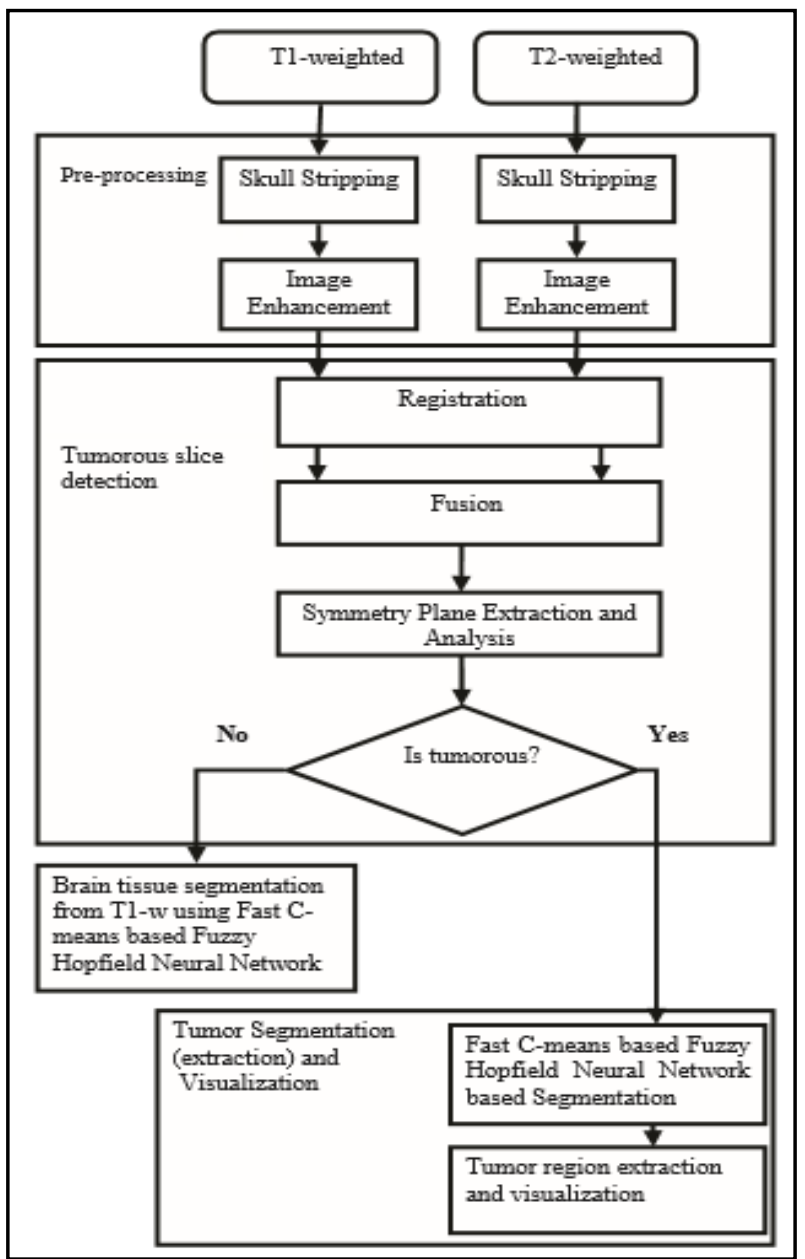

Fig 4: Flow of system [5]

\subsubsection{Tumorous Slice Detection}

The second component of the method based on: tumorous slice detection, it performs image registration, image fusion and symmetry plane extraction of MRI. In first step, Image registration is performed slice by slice basis. Then Image Fusion of the spatially aligned images is the next step. In third step, symmetry axis extraction is carried out on spatially aligned and fused MR images to identify the mid-sagittal plane of the brain. Finally, the histograms of images to the right and left of the mid-sagittal plane are analyzed to initially identify the existence of tumor.

In this paper, the abnormality of the brain depends on symmetry analysis of image gray levels. Brain tumors producing mass-effect displaces destroy the surrounding structures and infiltrating tumors affect tissue characteristics, changing the intensity levels in the image. These characteristics can provide important information that tumors may be present in brain.

\subsubsection{Tumor Segmentation and Visualization}

Here introduce Fuzzy C-means and Hopfield Neural Network. Then hybridization of the two algorithms is taken, called Fuzzy Hopfield Neural Network.

The FCM algorithm is a well known segmentation method based on minimizing an objective function. Clustering of brain tumor images, using Fuzzy C-means is robust and effective for finding similar types of tissues in the brain and 
localization. FCM shows good accuracy in performance and gives superior results in segmentation efficiency and convergence rate.

Hopfield Neural Network (HNN) derived from the well known neural network techniques, used for solving optimization issues based on the Lyapunov energy function. The classification result and its quality of classification primarily depend on weighting factors in the energy function. When the modified Lyapunov energy function is minimal, then the whole network becomes in a stable state. The final algorithm, Fuzzy Hopfield Neural Network (FHNN) is the combination of FCM and HNN method. Through this technique provides convenient online learning and parallel implementation for image segmentation. The objective function introduced by FHNN, and its minimization operations are done by modified Lyapunov energy function two dimensional fuzzy Hopfield neural networks. Optimal value detection of these weighting factors are very time consuming but FHNN can solve this problem easily.

\section{EXPERIMENTAL RESULTS AND DISCUSSIONS}

Here validating the proposed method using three different datasets. Those are, real tumorous brain MRI scans from Pioneer Diagnostic Center (PDC), real normal MR images from Internet Brain Segmentation Repository (IBSR), simulated tumor MR images. Also validating the performance using the most commonly used similarity metrics. Then validation is performed by comparing the proposed method's output with manually obtained ground truth. The inputs MR images apply to the skull stripping method and tested on IBSR and PDC datasets.

The tumorous slice classification result for PDC sets that compared with ground truth shown in the Figure 5. In which, sensitivity means the ability of a test to correctly identify those with the disease and test specificity is the ability of the test to correctly identify those without the disease. Here the true positive ratio for dataset is 0.9273 and true negative ratio is 0.8140 , so we can say that the method been used for evaluation process, accurately determines the patient with the tumor in comparison to the patient who does not have a tumor.

Among the total number of ground truth tumorous slices in PDC dataset only few slices are wrongly detected as false negative and among non-tumorous ground truth slices, some are wrongly detected as tumorous slices. Tumorous slice classification result for simulated tumor shown in Figure 6.

\begin{tabular}{|c|c|c|c|c|c|c|c|c|c|c|}
\hline \multirow{3}{*}{$\begin{array}{c}\text { Cases } \\
\text { Patient A }\end{array}$} & \multicolumn{2}{|c|}{$\begin{array}{l}\text { Ground } \\
\text { truth }\end{array}$} & \multicolumn{8}{|c|}{ Classification of slices by the automatic proposed method } \\
\hline & \multirow{2}{*}{ 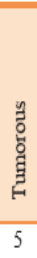 } & \multirow{2}{*}{ 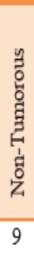 } & \multirow{2}{*}{$\begin{array}{l}\hat{A}^{2} \\
4\end{array}$} & \multirow{2}{*}{$\begin{array}{l}\vec{k} \\
4\end{array}$} & \multirow{2}{*}{$\begin{array}{l}1 \\
1\end{array}$} & \multirow{2}{*}{$\begin{array}{l}\mathrm{L}^{2} \\
5\end{array}$} & \multicolumn{2}{|c|}{$\begin{array}{c}\text { True positive } \\
\text { ratio } \\
\text { (Sensitivity) }\end{array}$} & \multicolumn{2}{|c|}{$\begin{array}{c}\text { True negative ratio } \\
\text { (Specificity) }\end{array}$} \\
\hline & & & & & & & $(4 / 5)$ & 0.8000 & $(4 / 9)$ & 0.4444 \\
\hline Patient B & 7 & 7 & 7 & 7 & 0 & 0 & $(7 / 7)$ & 1.0000 & $(7 / 7)$ & 1.0000 \\
\hline Patient C & 10 & 4 & 10 & 4 & 0 & 0 & $(10 / 10$ & 1.0000 & $(4 / 4)$ & 1.0000 \\
\hline patient D & 8 & 6 & 7 & 6 & 1 & 0 & $(7 / 8)$ & 0.8750 & $(6 / 6)$ & 1.0000 \\
\hline Patient $\mathrm{E}$ & 7 & 7 & 6 & 6 & 1 & 1 & $(6 / 7)$ & 0.8571 & $(6 / 7)$ & 0.8571 \\
\hline Patient $F$ & 9 & 5 & 8 & 4 & 1 & 1 & $(8 / 9)$ & 0.8889 & $(4 / 5)$ & 0.8000 \\
\hline Patient G & 9 & 5 & 9 & 4 & 0 & 1 & $(9 / 9)$ & 1.0000 & $(4 / 5)$ & 0.8000 \\
\hline Total & 55 & 43 & 51 & 35 & 4 & 8 & $(51 / 55$ & 0.9273 & $(35 / 43$ & 0.8140 \\
\hline
\end{tabular}

Fig 5: Tumorous slice classification result for PDC data sets [5]

\begin{tabular}{|c|c|c|c|c|c|c|c|c|}
\hline \multirow[b]{2}{*}{ Cases } & \multicolumn{2}{|c|}{$\begin{array}{l}\text { Ground } \\
\text { truth slices }\end{array}$} & \multicolumn{6}{|c|}{$\begin{array}{l}\text { Classification of slices by the automatic proposed } \\
\text { method }\end{array}$} \\
\hline & $\begin{array}{l}\text { 善 } \\
\text { 息 } \\
\text { 至 }\end{array}$ & 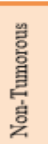 & $\leftrightarrow$ & $\pi$ & $\mathrm{k}^{2}$ & $k^{2}$ & $\begin{array}{l}\text { (True } \\
\text { positive } \\
\text { ratio) } \\
\text { sensitivity }\end{array}$ & $\begin{array}{c}\text { (True } \\
\text { negative } \\
\text { ratio) } \\
\text { specificity }\end{array}$ \\
\hline S01 & 49 & 21 & 49 & 8 & 0 & 13 & $\begin{array}{l}(49 / 49) \\
1.0000\end{array}$ & $\begin{array}{l}(8 / 21) \\
0.3810\end{array}$ \\
\hline S02 & 31 & 39 & 31 & 31 & 0 & 8 & $\begin{array}{l}(31 / 31) \\
1.0000\end{array}$ & $\begin{array}{l}(31 / 39) \\
0.7949\end{array}$ \\
\hline S03 & 30 & 40 & 29 & 40 & 1 & 0 & $\begin{array}{l}(29 / 30) \\
0.9667\end{array}$ & $\begin{array}{l}(40 / 40) \\
1.0000\end{array}$ \\
\hline S04 & 36 & 34 & 26 & 30 & 10 & 4 & $\begin{array}{l}(26 / 36) \\
0.7222\end{array}$ & $\begin{array}{l}(30 / 34) \\
0.8824\end{array}$ \\
\hline S05 & 50 & 20 & 41 & 13 & 9 & 7 & $\begin{array}{l}(41 / 50) \\
0.8200\end{array}$ & $\begin{array}{l}(13 / 20) \\
0.6500\end{array}$ \\
\hline S06 & 22 & 48 & 17 & 48 & 5 & 0 & $\begin{array}{l}(17 / 22) \\
0.7727\end{array}$ & $\begin{array}{l}(48 / 48) \\
1.0000\end{array}$ \\
\hline S07 & 48 & 22 & 47 & 15 & 1 & 7 & $\begin{array}{l}(47 / 48) \\
0.9792\end{array}$ & $\begin{array}{l}(15 / 22) \\
0.6818\end{array}$ \\
\hline S08 & 36 & 34 & 0 & 34 & 36 & 0 & $\begin{array}{l}(0 / 36) \\
0.0000\end{array}$ & $\begin{array}{l}(34 / 34) \\
1.0000\end{array}$ \\
\hline S09 & 51 & 19 & 51 & 10 & 0 & 9 & $\begin{array}{l}(51 / 51) \\
1.0000\end{array}$ & $\begin{array}{l}(10 / 19) \\
0.5263\end{array}$ \\
\hline Total & 353 & 277 & 291 & 229 & 62 & 48 & $\begin{array}{c}(291 / 353) \\
0.8244\end{array}$ & $\begin{array}{l}(229 / 277) \\
0.8267\end{array}$ \\
\hline 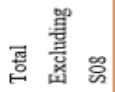 & 317 & 243 & 291 & 195 & 26 & 48 & $\begin{array}{c}(291 / 317) \\
0.9180\end{array}$ & $\begin{array}{c}(195 / 243) \\
0.8025\end{array}$ \\
\hline
\end{tabular}

Fig 6: Tumorous slice classification result for simulated tumor datasets [5]

The result obtained for simulated brain tumor dataset is with true positive ratio (sensitivity) of 0.8244 and true negative ratio (specificity) of 0.8267 . But, excluding S08 dataset, where symmetry based tumorous slice detection fails, the true positive ratio (sensitivity) reaches 0.9180 and true negative ratio (specificity) becomes 0.8025 as shown on Figure 6 .

Figure 7 and Figure 8 shows the sample segmentation results on simulated tumor and PDC datasets. Results of nontumorous slices brain tissue segmentation of simulated datasets shown in Figure 9. 

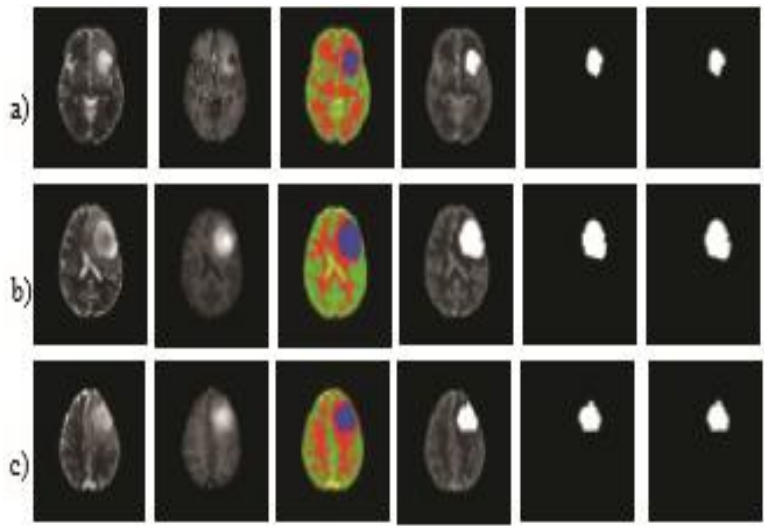

Fig 7: Sample results of tumor segmentation and extraction on S01 simulated tumor dataset. Rows from left to right: a, b, c respectively: T2-w image, T1-w image, segmented image by the system, ground truth segmented tumor, extracted tumor by the proposed method and extracted ground truth tumor region [5]
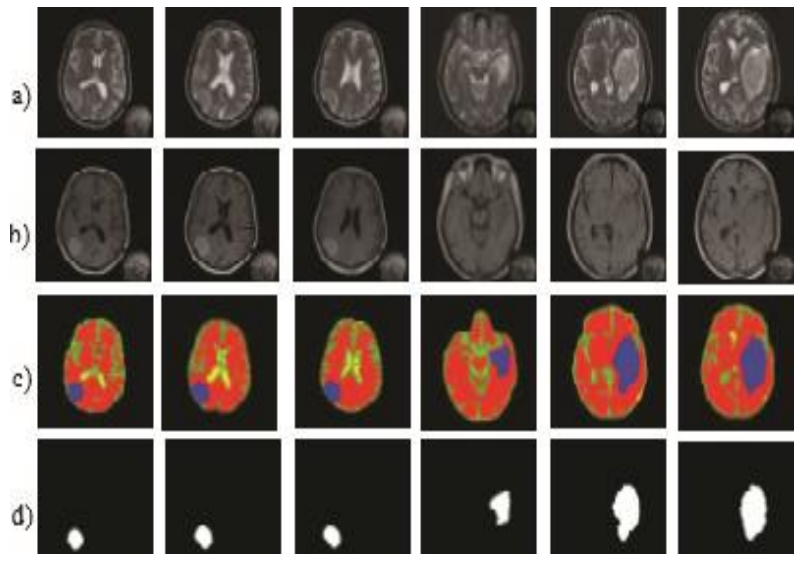

c)
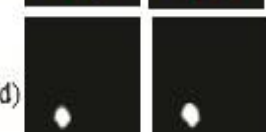

Fig 8: Result of tumor segmentation and extraction on PDC datasets. Columns from left to right Patient A. a) T2w image, b) T1-w image, c) segmented image by the system and d) extracted tumor from segmented image; and the

last three columns represent Patient B's scans [5]

The result shows the effectiveness of the hybrid method in segmenting brain tumors and normal brain tissues in brain MR images. But it has some difficulties in segmenting brain tissues in low tissue contrast MR images. To overcome this issue we can apply GMM in input MR images to improve the visual quality. It will automatically improve brightness, content and contrast of a gray level input image.

\subsection{Pros and cons}

It does not need training data. Second it is capable to segment normal tissues and both low and high contrast image enhancement using GMM. Thirdly, it can be implemented in real time application due to high speed segmentation capability. Finally, the proposed method is highly automatic in that no user intervention is required to produce normal tissues and tumor segmentation results in a limited time. a)
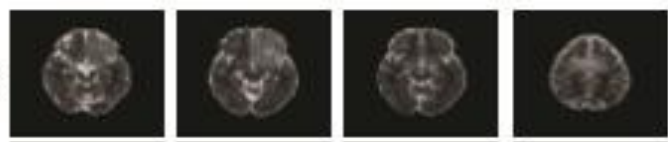

b)
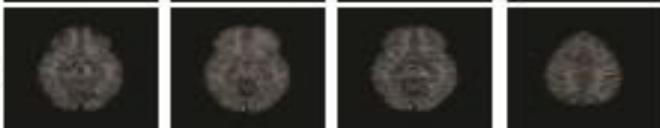

c)
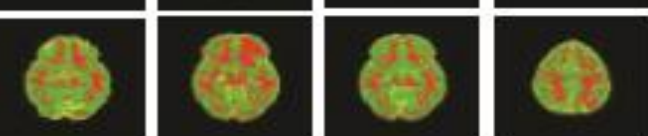

d)
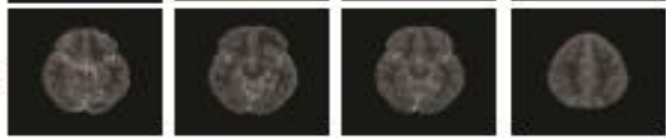

Fig 9: Result of non-tumorous slices brain tissue segmentation of simulated datasets a) T2-w image, b) T1$w$ image, c) segmented image by the proposed system and d) ground truth tissue segmentation [5]

Table 1: Summary of brain tumor segmentation methods

\begin{tabular}{|c|c|c|}
\hline $\begin{array}{l}\text { Segmentation } \\
\text { method }\end{array}$ & Merits & Demerits \\
\hline Threshold based & $\begin{array}{c}\text { Simpler, fast } \\
\text { computations and } \\
\text { lower complexity }\end{array}$ & \begin{tabular}{|c|} 
Uncertainty, \\
accuracy problem, \\
Tumor area \\
enhancement \\
limited
\end{tabular} \\
\hline Region based method & $\begin{array}{l}\text { Correctly segments } \\
\text { regions that have } \\
\text { similar properties, } \\
\text { produces connected } \\
\text { region }\end{array}$ & $\begin{array}{c}\text { Expensive in terms } \\
\text { of computation of } \\
\text { both time and } \\
\text { memory }\end{array}$ \\
\hline $\begin{array}{c}\text { Fuzzy c means } \\
\text { method }\end{array}$ & $\begin{array}{l}\text { Better accuracy, It } \\
\text { converges the tumor } \\
\text { boundaries }\end{array}$ & $\begin{array}{c}\text { Long computational } \\
\text { time, sensitivity to } \\
\text { noise }\end{array}$ \\
\hline $\begin{array}{l}\text { K-Mean clustering is } \\
\text { followed by FCM }\end{array}$ & $\begin{array}{l}\text { Time, accuracy, } \\
\text { iterations have been } \\
\text { the major focus }\end{array}$ & \begin{tabular}{|c|} 
FCM generates \\
overlapping results, \\
issues on segment \\
colored images
\end{tabular} \\
\hline K-means algorithm & $\begin{array}{l}\text { Median filtering and } \\
\text { morphological } \\
\text { operation }\end{array}$ & $\begin{array}{c}\text { Has ignored the } \\
\text { poor quality images }\end{array}$ \\
\hline $\begin{array}{c}\text { Hybrid method using } \\
\text { FHNN }\end{array}$ & $\begin{array}{l}\text { High speed } \\
\text { segmentation, } \\
\text { limited time, } \\
\text { accuracy }\end{array}$ & $\begin{array}{c}\text { Low tissue contrast } \\
\text { MR images }\end{array}$ \\
\hline $\begin{array}{c}\text { FHNN method } \\
\text { followed by GMM } \\
\text { technique }\end{array}$ & $\begin{array}{c}\text { Low contrast } \\
\text { enhancement, high } \\
\text { accuracy and speed }\end{array}$ & - \\
\hline
\end{tabular}

The above Table 1 is a summary of some relatively good algorithms of MRI-based brain tumor segmentation. The segmentation methods merits and demerits are also mentioned 
in the respective table. Compared to other efficient algorithm, hybrid method of FHNN shows more accuracy and improves the visual quality with the support of GMM.

\section{RECOMMENDATION AND CONCLUSION}

In this paper, presented some of the efficient research works done on brain tumor detection. Different techniques for feature extraction and segmentation have been discussed here. Each technique has got its advantages and limitations. From Literature survey, it is concluded that the hybrid method of FHNN technique is the one of the most effective methods for detection and segmentation of brain tumor. And also we can propose the GMM technique to improve the visual quality of the images in hybrid method. GMM is mainly concentrated on the low contrast enhancement of an image. In which, a step by step procedure for an automatic brain tumor detection and segmentation using fuzzy Hopfield neural network is presented. In the future, it can be implemented as real time application due to the high speed segmentation ability of the fuzzy Hopfield neural network. The method is a combination of different techniques and composed of three modules. The performance of hybrid method can be measured on various MRI scans and the method is validated against ground truth using commonly used validation metrics. Compared to other segmentation method, hybrid method of FHNN technique shows good performance in case of accuracy, speed and time. The proposed GMM is focused only on getting better enhancement of the image and it shows satisfactory results.

\section{REFERENCES}

[1] Automated Brain Tumor Segmentation Using Region Growing Algorithm by Extracting Feature Volume 3, Issue 12, December 2014, ShraddhaP. Dhumalland Ashwini S Gaikwad.

[2] Proceedings of the UGC Sponsored National Conference on Advanced Networking and Applications,27th March 2015 "Brain Tumor Detection and Identification Using K-Means Clustering Technique“ Malathi R Department of Computer Science.

[3] Preetha, R., and G. R. Suresh. "Performance Analysis of Fuzzy C Means Algorithm in Automated Detection of
Brain Tumor." In Computing and Communication Technologies (WCCCT), 2014 World Congress on, pp. 3033. IEEE, 2014.

[4] Abdel-Maksoud, Eman, Mohammed Elmogy, and Rashid Al-Awadi. "Brain tumor segmentation based on a hybrid clustering technique." Egyptian Informatics Journal (2015)

[5] Yehualashet Megersa, Electrical and Computer Engineering Department, Addis Ababa University, "Brain Tumour Detection and segmentation using Hybrid Intelligent Algorithm", November 2015.

[6] "Image Contrast Enhancement Using Guassian Mixture Modeling And Its Comparison With Different Algorithms" Sabahat Fatima1, Dr. Shubhang and Hashmath Fatima3.International Journal Of Advancement In Engineering Technology,Volume 3, Issue 2 May 2016.

[7] Dvorak, Pavel, Walter Kropatsch, and Karel Bartusek. "Automatic detection of brain tumors in MR images."In Telecommunications and Signal Processing (TSP), 2013 36th International Conference on, pp. 577-580. IEEE, 2013.

[8] Parisot,Sarah,HuguesDuffau,StéphaneChemouny, and Nikos Paragios."Graph-based detection, segmentation \& characterization of brain tumors." In Computer Vision and Pattern Recognition (CVPR), 2012 IEEE Conference on, pp. 988-995. IEEE, 2012

[9] Sonu suhag, L. M. SAINI, "Automatic Detection Of Brain Tumor By Image Processing In Matlab", SARCIRF international conference, 24th may-2015

[10] Dvorak, Pavel, Walter Kropatsch, and Karel Bartusek. "Automatic detection of brain tumors in MR images." In Telecommunications and Signal Processing (TSP), 2013 36th International Conference on, pp. 577-580. IEEE, 2013.

[11] Ferlay J, Shin HR, Bray F, Forman D, Mathers C and Parkin DM, 'GLOBOCAN 2008 v2.0, Cancer Incidence and Mortality Worldwide', International Agency for Research on Cancer, Lyon, France, 2010. 